\title{
Capital humano: una visión desde la teoría crítica
}

\author{
Human capital: a vision from critical theory \\ Capital humano: uma visão desde a perspectiva da teoria crítica \\ Duvan Emilio Ramírez Ospina ${ }^{1}$ \\ Universidad de Manizales / Facultad de Ciencias Contables, Económicas y Administrativas, Colombia \\ Universidad Simón Bolívar, Colombia
}

\begin{abstract}
Resumen
Este trabajo es el resultado de la investigación Capital Humano como factor de crecimiento Económico, en el cual se desarrolla una reflexión crítica sobre la teoría del Capital Humano, el abordaje se hace desde la teoría económica y el análisis tiene como referente los planteamientos de la Escuela de Frankfurt, especialmente en lo que tiene que ver con el uso desde la perspectiva de la racionalidad. Desde el punto de vista metodológico se trata de una investigación cualitativa, basada en un proceso de carácter interpretativo y comprensivo de tipo Histórico Hermenéutico, el método utilizado responde a una finalidad de descripción, interpretación, argumentación, que permitan avanzar hacia la comprensión de las temáticas estudiadas en un proceso dialéctico. Como resultado del proceso investigativo se hace un análisis de la instrumentalización de la educación, la formación, la capacidad de trabajo y el estado de salud del hombre, y aún de su propio ser, las cuales se consideran de la misma naturaleza que una maquina y quedan cosificadas al ser convertidas en mercancías comerciales que se venden en el mercado, lo que determina la posibilidad de colocarle un precio pagado en el mercado a la productividad de un tipo de trabajo determinado, a la acción del propio hombre y el desarrollo de sus capacidades superiores que deberían permitirle contribuir al logro de una sociedad mejor y una vida más digna.
\end{abstract}

Palabras-clave: Capital Humano. Teoría Crítica. Racionalidad Instrumental. Crecimiento Económico. Educación.

\begin{abstract}
This work is the result of human capital research as an economic growth factor, in which it is developed a critical reflection on human capital theory, it is addressed from the economic theory and the analysis has as a reference the approaches from the Frankfurt School, especially in what has to do with the use from perspective of rationality. From the methodological point of view it is a qualitative research, based on a process of interpretive and hermeneutic understanding of historical type, the method responds to an objective description, interpretation, argument for moving towards the understanding of the thematic studied in a dialectical process. As a result of the research process an analysis of the instrumentalization of education, training, work capacity and health status of man is made, and even of your own self, which are considered of the same nature as a machine and are reified to be converted into commercial goods sold in the market, which determines the possibility of placing a paid market productivity of a particular type of work, human action itself and the development of their superior capacities that should allow contribute to achieve a better society and a better life.
\end{abstract}

Keywords: Human capital. Critical Theory. Instrumental Rationality. Economic Growth. Education.

Artículo enviado en 17 de enero de 2014 y aprobado para publicación en 29 de julio de 2014.

DOI: http://dx.doi.org/10.1590/1679-395114754

1 Doctor en Administración por la Universidad Andina Simón Bolívar sede Ecuador; Magister en gerencia del talento Humano; Especialista en Mercadeo; Economista. Decano Facultad de Ciencias Contables, Económicas y Administrativas de la Universidad de Manizales; Profesor de Posgrados Universidad Simón Bolívar. Dirección: Carrera 9 No 1903 Manizales, Colombia. E-mail: duvramirez@hotmail.com 


\section{Resumo}

Este trabalho é o resultado da pesquisa Capital Humano como fator de crescimento Econômico, no qual se desenvolve uma reflexão crítica sobre a teoria do Capital humano, a abordagem se faz desde a perspectiva da teoria econômica e a analise têm como referente às abordagens da Escola de Frankfurt, especialmente no que diz a respeito ao uso desde a perspectiva da racionalidade. A partir do ponto de vista metodológico trata-se de uma pesquisa qualitativa, baseada em um processo de caráter interpretativo e compreensivo de tipo Histórico Hermenêutico, o método utilizado responde a finalidade de descrição, interpretação, argumentação que permitam avançar em direção de uma compreensão das temáticas estudadas em um processo dialético. Como resultado do processo de pesquisa se faz uma análise da instrumentalização da educação, a formação, a capacidade de trabalho e o estado de saúde do homem, e até mesmo o seu próprio ser, que são considerados da mesma natureza de uma máquina que se torna objeto ao serem convertidas em bens de comerciais que se vendem no mercado, o que determina a possibilidade de colocar um preço no mercado à produtividade de um tipo de trabalho determinado, à ação do próprio homem e o desenvolvimento de suas capacidades superiores que deveriam permiti-lhe contribuir para uma sociedade melhor e mais digna.

Palavras-chave: Capital Humano. Teoria Crítica. Racionalidade Instrumental. Crescimento Econômico. Educação.

\section{Introducción}

Este trabajo es el resultado de la investigación Capital Humano como factor de crecimiento Económico, en el cual se desarrolla una reflexión crítica sobre la teoría del Capital Humano, que se ha convertido en un tema recurrente en los discursos de los dirigentes empresariales y cócteles de gerentes, en los cuales se plantea la importancia de la gente en los diferentes procesos de la organización, según esta visión el recurso más importante de las organizaciones es su capital humano; esta visión tiene dos problemas fundamentales, de un lado, se reduce el ser humano a la simple condición de recurso, que se usa y se desecha cuando ha cumplido el fin para el cual ha sido adquirido.

De otro lado, este tipo de afirmaciones no corresponden con los procesos de toma de decisiones en las organizaciones; generalmente, cuando se presenta una situación de crisis, bien sea originada en problemas de demanda o de incremento de los niveles de competencia, la primera decisión de esos gerentes que antes pregonaban en discursos y cócteles sobre la importancia de su gente como factor estratégico de alta competitividad, está asociada al despido masivo del que antes denominaba el factor más importante de la organización.

De acuerdo con este planteamiento, se aborda el estudio del concepto de capital humano teniendo como referente los desarrollos de la teoría económica, desde los primeros clásicos hasta los autores contemporáneos que han efectuado sus trabajos teóricos alrededor del tema, entre los cuales se encuentran distintos premios Nobel de economía. El abordaje desde la teoría crítica parte de la Escuela de Frankfurt, teniendo en cuenta los planteamientos de Adorno, Horkheimer y Habermas; además fueron considerados los escritos de Marx y Weber, entre otros.

En este documento no se trata de discutir la importancia de la salud, la formación para el trabajo y la educación en los procesos de producción; sino más bien, de analizar la instrumentalización que se hace de la educación, la formación, la capacidad de trabajo y el estado de salud del hombre, y aún de su propio ser, las cuales se consideran de la misma naturaleza que una maquina y quedan cosificadas al ser convertidas en mercancías comerciales que se venden en el mercado, lo que determina la posibilidad de colocarle un precio a la productividad de un tipo de trabajo determinado, a la acción del propio hombre y el desarrollo de sus capacidades superiores que deberían permitirle contribuir al logro de una sociedad mejor. 


\section{Visión Crítica De La Teoria Del Capital Humano}

Desde sus inicios, la teoría económica ha señalado la importancia de las personas para lograr unos mayores niveles de producción, es así como Smith (1983), representante de la Escuela Clásica, identificó la importancia de la formación para lograr mejores resultados en el trabajo, en la parte I del primer tomo de La Riqueza de las Naciones, refiriéndose a las desigualdades que resultan de la naturaleza de los empleos, reconoce cinco causas que influyen para que en unos empleos sea mayor que en otros la ganancia pecuniaria.

La segunda causa mencionada por Smith es, "la facilidad y poco coste, o la dificultad y gastos para aprenderlos"; en la sustentación de este planteamiento compara a un hombre educado, con una costosa máquina, y afirma: "Un hombre educado a expensas de mucho trabajo y tiempo, en cualquiera de aquellos oficios que requieren una destreza y pericia extraordinaria, debe compararse a una de estas costosas máquinas......" de esta forma, Smith, iguala la educación y la formación para el trabajo con la categoría capital, lo cual debe permitirle a su poseedor recibir una renta más alta en la medida que las capacidades superiores resultantes del proceso de formación contribuyan al logro de un mayor producto, si el dueño de la máquina recibe una renta por el capital invertido en ella; de la misma forma el dueño del conocimiento o la pericia extraordinaria, debería recibir una renta por el capital invertido en el proceso de adquisición de esas capacidades y pericias extraordinarias.

En esta perspectiva, las actividades que no sean útiles o que no sirven para mantener el aparato productivo en funcionamiento y en un proceso constante de crecimiento de sus ganancias son señaladas como carentes de sentido o superfluas;sin embargo, según el planteamiento de Horkheimer (2002) "el trabajo productivo, sea manual o intelectual, se ha vuelto honorable, de hecho, es el único modo aceptado de pasar la vida, y cualquier ocupación, cualquier persecución de un objetivo que conlleve finalmente un ingreso es caracterizada como productiva.

En este sentido se puede señalar la perdida de la posibilidad de realización del individuo por medio del trabajo, al convertirse este en una actividad mercantil, necesaria para poder obtener los medios de subsistencia y que contribuye a transformar el dinero en capital, de acuerdo con el planteamiento de Marx(1991),segúnel cual, el poseedor de dinero para convertirlo en capital tiene que encontrar en el mercado el obrero libre en dos sentidos; de una parte, ha de disponer libremente de su fuerza de trabajo como mercancía y, de otra parte, no ha de tener otras mercancías que ofrecer en venta. Este estado de cosas es el fruto de un desarrollo histórico precedente, el producto de una serie larga de transformaciones más antiguas en el campo de la producción social.

En la existencia del producto como mercancía van implícitas condiciones históricas determinadas; para que el producto se convierta en mercancía, es necesario que no sea creado como medio directo de subsistencia para el productor, lo cual acontece con el trabajo en el sistema capitalista y con las habilidades especiales que adquiere el trabajador por medio de los diferentes procesos de educación y formación para el trabajo; en este sistema, la educación y la formación, no están orientadas a contribuir con el logro de mayores niveles de libertad para los individuos, sino que están orientadas a prestar servicios productivos que los trabajadores venden en el mercado y las organizaciones compran.

Por lo cual, la producción que resulte de la adquisición de habilidades y conocimientos como consecuencia del proceso educativo, no son consumidas por su productor, sino que son vendidas en el mercado, convirtiéndose en una mercancía; la transformación del trabajo en mercancía lleva consigo, una división social del trabajo, tan desarrollada que en ella se consuma el divorcio entre el valor de uso y el valor de cambio; entendido aquel como la posibilidad que tiene un bien de satisfacer una necesidad del ser humano; mientras que el valor de cambio, es la propiedad por medio de la cual un bien se puede vender en un mercado; y es el resultado de tener incorporado trabajo del hombre que ha sido socialmente acumulado a lo largo de un proceso histórico dedesarrollo de las diferentes formas de producción.

La división social del trabajo es condición para la existencia de la producción de mercancías, aunque la producción de mercancías no es, condición para la existencia de la división social del trabajo. Esto encierra 
una gran contradicción; de un lado, el valor de cambio es el fruto del trabajo del hombre que se ha logrado acumular, pero al mismo tiempo, el trabajo del hombre por si mismo se convierte en valor de cambio al ser comprado y vendido en el mercado (MARX, 1991).

De esta forma, se puede observar que en el valor de uso de toda mercancía hay una determinada actividad productiva según fines establecidos, un trabajo útil. Los valores de uso no pueden enfrentarse como mercancías si no hay en ellos trabajos útiles cualitativamente diversos; en consecuencia, las capacidades desarrolladas por medio de la educación y el entrenamiento para el trabajo en su condición de valor de uso útil para el empresario, se enfrenta a los valores de uso de los productos que el trabajador cualquiera sea su condición requiere para satisfacer sus necesidades y las de su familia, lo cual permite consumar la transformación de la capacidad de trabajo en una mercancía que se intercambia en el mercado bajo el mismo sistema de intercambio de otras mercancías.

El comprador de fuerza de trabajo, o sea el capitalista, la consume haciendo trabajar al que se la ha vendido, el obrero; para convertirla en fuerza de trabajo en acción.Para poder convertir el trabajo en mercancía, tiene que convertirlo en valores de uso, en cosas que sirvan para satisfacer necesidades de cualquier tipo; así, lo que el capitalista hace ejecutar al obrero es un determinado valor de uso, que pasa a ser propiedad del capitalista, que ha pagado por la fuerza de trabajo, lo mismo que ha pagado por cualquier otra mercancía necesaria para el proceso de producción, incluyendo las maquinas, caso en el cual se puede ejecutar la comparación establecida por Smith (1983)entre el trabajador calificado y la costosa máquina.

El desarrollo del trabajo es un proceso entre cosas que el capitalista ha comprado, entre cosas que le pertenecen. Por eso el producto de ese proceso le pertenece exactamente igual que el proceso de transformación de todos las demás mercancías que ha comprado para poner en marcha el proceso de producción, en este caso, la única diferencia entre un trabajo superior considerado así por la necesidad de invertir una mayor cantidad en su producción como sería el caso del trabajo especializado, frente al trabajo medio, estaría determinada por su objetivización en valores relativamente superiores, que implican lo que Smith señalaba como mayor ganancia pecuniaria.

Marx (1991), al igual que Smith (1983), reconoce la importancia de la división del trabajo en el proceso de acumulación de capital, pero le agrega un ingrediente nuevo; la necesidad de que en el mercado se produzca un encuentro entre el poseedor de dinero y alguien que sólo posee su fuerza de trabajo para convertirla en mercancía, que pueda cambiar por dinero, para poder adquirir otras mercancías, que requiere para su subsistencia y la de su familia, siendo este otro elemento fundamental de la instrumentalización de la capacidad de trabajo del ser humano que se ve objetivada bajo la expresión capital humano, término que fue acuñado por Schultz (1985).

La instrumentalización de las capacidades superiores de los seres humanos se ven ratificadas en el planteamiento de Lewis (1976), quien analizó un doble problema planteado por la educación; por un lado, representa un servicio de consumo y de otro un servicio de inversión; en la medida que es una inversión contribuye directamente al incremento de la producción; el dinero gastado en educación es una inversión de capital, de la misma naturaleza que el dinero gastado en obras de riego; pero sólo cuando este contribuye de manera directa al proceso de producción de bienes y servicios orientados al mercado.

Además, consideró como un problema, la dificultad para señalar los límites de la clase de educación que contribuye más al disfrute de la vida que a la producción; en este sentido, dijo que algunos miembros de la comunidad deben saber leer y escribir, puesto que, de otra forma, no podrían desempeñar bien su trabajo. Pero no puede afirmarse que la productividad de la mayoría de los campesinos, porteros, barberos, o sirvientes domésticos aumentará, si se les enseña a leer y escribir; por lo tanto, la educación de estos grupos es deseable no como una inversión, sino como un bien de consumo, porque se supone que les ayudará a disfrutar mejor algunas cosas o a comprender mejor otras, no implicando esto que sean más felices, pero si más humanos. 
Este pensamiento de Lewis, puede asimilarse a lo que Horkheimer (2002) denomina como una razón subjetiva, que tiene que ver esencialmente con medios y fines, con la adecuación de los métodos y modos de proceder a los fines, unos fines que son más o menos asumidos y que presuntamente se sobreentienden; desde este punto de vista, el desarrollo de las capacidades intelectuales de los seres humanos, sólo se justifican en la medida que se conviertan en un método o modo que contribuye a lograr el fin de incrementar la productividad y las ganancias, a mejorar los proceso de producción e incorporarse en los productos que son llevados al mercado de acuerdo con unas necesidades determinadas; considerando que todos aquellos procesos de formación que no se enmarquen en esta lógica de medios, métodos y fines son similares a otros gastos considerados como consumo, sin tener en cuenta que estos gastos, también pueden tener la importancia de una inversión en la medida que se evalué desde una visión opuesta de la razón, vista como una fuerza no sólo de la conciencia individual, sino también en el mundo objetivo en las relaciones entre los hombres y entre las clases sociales, en las instituciones sociales, en la naturaleza y en sus manifestaciones; en la que se considera, no sólo el ser humano y sus fines, sino la totalidad englobante de todos los entes.

Desde el punto de vista económico, Lewis (1976), la educación que no representa una inversión productiva equivalente a otros bienes de consumo, como lo son los vestidos, las casas, los alimentos. La educación universitaria es considerada como una inversión por quienes se esfuerzan en obtenerla; es un medio para alcanzar un rango social más alto y un ingreso mayor, en este punto aparece de nuevo la importancia de los fines. En países que disponen de grandes servicios universitarios, si al mismo tiempo no se produce el crecimiento y el desarrollo económico y no existe un mercado en expansión para los profesionales formados, el país se ve inundado de personas que han hecho estudios superiores y tienen que aceptar los trabajos que buenamente puedan obtener, por lo que se sienten en extremo descontentos y constituyen material de primera clase para la agitación política, pues no están de acuerdo con los salarios que obtienen o aún con la posición social que creen merecer en atención a sus estudios superiores.

Desde esta perspectiva, la educación y la formación son consideradas como elementos que deben contribuir al logro de objetivos meramente económicos, sin importar sus efectos en la realización de los individuos y en el incremento de sus posibilidades de obtener mayores grados de libertad; sólo son importantes en la medida que contribuyen al cumplimiento de un fin económico, asimilándolas a las diferentes formas de inversión en máquinas y equipos señalada por Smith (1983).

Vistas de esta forma, las decisiones de los individuos sobre profesión se convierten en lo que Habermas (2000) llama una tarea pragmática que se resuelve como un caso sencillo, similar a los problemas prácticos que se resuelven acudiendo a fundamentos en los que se apoyan decisiones de tipo racional, entre las distintas posibilidades de acción a la vista de un problema que es necesario resolver si se desea alcanzar un determinado objetivo; lo cual depende en primera instancia de las preferencias y de las opciones que están abiertas en cada situación, llevando a la búsqueda de los fundamentos para una decisión racional; que por ser consideradas triviales o débiles no necesitan fundamentación alguna.

Por el contrario, teniendo en cuenta la perspectiva de Habermas (2000), sobre la elección de una profesión considerada una decisión compleja que debe ir unida a la pregunta por las inclinaciones o a cerca de que le interesa a uno ser; lo que significa: qué tipo de persona se es y al mismo tiempo que tipo de persona le gustaría ser, por lo tanto, esto no debe tratarse como una decisión sencilla, sino todo lo contrario, como una decisión compleja, que no puede ser abordada como una decisión pragmática, toda vez que implica decidir sobre el tipo de vida que se quiere llevar; implicando valoraciones que afectan no sólo a disposiciones e inclinaciones casuales, sino a la autocomprensión de una persona, al tipo de vida que lleva, su carácter: todas ellas entretejidas con la propia identidad de las personas.

Por lo tanto, la educación no puede verse como un simple proceso que contribuye a incrementar el capital de una persona para ser puesto al servicio de una actividad productiva, o como un bien de consumo cuando no es posible vincularlo al proceso de producción; por lo tanto, a la corriente de explotación del capital por parte de los empresarios; en este orden de ideas, la educación debe verse como una posibilidad de autocomprensión que apoya un proyecto de vida y que debe estar orientado de acuerdo con lo que la persona 
es y lo que desea ser, en consecuencia es una decisión que no debe consultar los mecanismos de mercado del sistema capitalista.

Continuando con los desarrollos teóricos de la economía que asimilan las capacidades humanas al capital, han permitido abordar desde diferentes perspectivas la formación y capacitación en y para el trabajo, resaltando la importancia de este factor en la actividad económica. Es así, como dentro de la Escuela Marginalista, entre quienes trabajaron la teoría del equilibrio se destaca Walras (1987), quien plantea:

Se suponen insertos en el sistema económico tres categorías de sujetos, distintos según la naturaleza de los bienes de capital a su disposición. Serán terratenientes, que poseen la tierra, trabajadores, que poseen capital personal y capitalistas en sentido estricto, que poseen los bienes de capital propiamente dichos. Cada uno de estos sujetos, en cuanto propietarios de capital, están en condiciones de ofrecer al mercado los servicios productivos de sus capitales".

De esta forma, Walras (1987) considera bajo la misma condición la posesión de capital físico o capital personal, que en este caso se puede asimilar al concepto que aparece años después de Capital humano.

En este planteamiento de Walras (1987) que parece tener la intención de igualar la posesión de capital físico dedicado a procesos de producción, con la fuerza de trabajo, que denomina capital personal; se oculta el sentido de la acción orientada hacia el lucro identificada por Weber (2003) como el capitalismo racional, dedicado a la búsqueda de ganancia mediante la organización racional del trabajo y el intercambio mercantil, que presupone tanto la consolidación del mercado, como la apropiación por parte del empresario de los instrumentos de trabajo, las fuentes de energía y las materias primas; lo cual implica la ausencia completa de apropiación por parte de los trabajadores tanto de los puestos de trabajo como de las propiedades lucrativas y, al contrario, ausencia de apropiación de los trabajadores por el propietario, como factor fundamental del trabajo libre, libertad en el mercado de trabajo y libertad en la selección de los trabajadores; lo cual se encuentra relacionado con la misma libertad que Marx (1991) había señalado como necesaria para que el capitalista pueda convertir su dinero en capital.

De la teoría clásica de la economía, se deriva la escuela Marginalista y de esta surge la teoría del capital y el crecimiento que da origen a la escuela del crecimiento, cuyo primer exponente es Schultz (1985) quien refiriéndose al miedo que los economistas tienen al concepto de inversión en hombres, afirma:

"Son pocos los que han considerado a los seres humanos como capital, pero entre ellos hay tres nombres distinguidos. Adam Smith, filósofo y economista, incluyó como parte del capital todas las capacidades adquiridas y útiles de todos los habitantes de una nación. Así lo hizo también H. Von Thunen (1966), quien añadió que la aplicación del concepto de capital al hombre ni le degrada ni estorba su libertad y dignidad; antes al contrario, la falta de aplicación de ese concepto resultaba especialmente perniciosa en las guerras; pues en ellas... con tal de salvar un cañón se sacrifica, sin pensarlo dos veces en una batalla, a cien seres humanos que se encuentran en la plenitud de sus vidas". El motivo es que la compra de un cañón produce un desembolso de fondos públicos, mientras que los seres humanos se obtienen gratis mediante un decreto de alistamiento"

En los planteamientos de Shcultz (1985), se encuentra en esencia lo que Weber (2003) denomina el espíritu del capitalismo, en el cual todo es susceptible de ser convertido en dinero, y este a su vez se podría convertir en capital de acuerdo con la fórmula de Marx (1991)cuando el poseedor de dinero puede encontrar en el mercado al obrero libre para vender su fuerza de trabajo y libre de medios de producción para obtener los medios de subsistencia.

En esta perspectiva; se piensa que el tiempo es dinero, así, el tiempo dedicado al ocio es una perdida monetaria, representada por la cantidad dejada de ganar mientras se dedica a una acción diferente de 
producir; el crédito es dinero, si un acreedor deja en manos de su deudor por largo tiempo su dinero, le está permitiendo ganar un interés y otros beneficios de conservar este medio de liquidez; el dinero es fértil y productivo, el dinero puede producir dinero, la descendencia puede producir todavía más y así sucesivamente, cuanto más dinero hay, más produce al ser invertido, de modo que el provecho aumenta rápidamente sin cesar. La moralidad es útil porque proporciona crédito y este produce dinero, lo mismo ocurre con la puntualidad, la diligencia, la moderación, la apariencia de honradez; en resumen todo debe producir dinero.

De esta forma, en una versión renovada del espíritu del capitalismo, se considera capital al hombre con conocimientos, habilidades y un estado de salud apropiado para poder participar en los procesos de producción, generar dinero y ganancias. Esto es mucho más importante si se considera que en esta versión del capitalismo, la ganancia no se tiene como un medio para satisfacer las necesidades vitales materiales del hombre; sino más bien, como un fin la vida del ser humano, de esta forma, la aparición del concepto de capital humano como fruto de un desarrollo histórico de la teoría económica, se puede plantear como una materialización del espíritu del capitalismo, en el sentido de que todo es dinero y el dinero se convierte en capital, lo que implica que el mismo hombre es sujeto de convertirse en dinero y por lo tanto en capital.Esta forma de ver todo como susceptible de ser convertido en dinero y este en capital, ha llevado a la sociedad capitalista al desenfreno absoluto yconsiente de la voluntad de lucrarse, al aplicarse esta voluntad a los seres humanos considerados como capital, lleva al empresario a la búsqueda de obtener de los trabajadores el rendimiento máximo posible, sin importar si estos trabajan con el conocimiento o con la fuerza física.

La consecuencia de esta forma de ver a los hombres como simples productores de dinero y como capital, se ve reflejada en los planteamientos de Adorno y Horkheimer (2007) cuando afirman que la técnica es la esencia del saber. Este no aspira a conceptos e imágenes, tampoco a la felicidad del conocimiento, sino al método, a la explotación del trabajo de los otros, al capital. Lo que los hombres quieren de la naturaleza es servirse de ella para dominarla por completo, a ella y a los hombres, ninguna otra cosa cuenta, sin importar la satisfacción de la verdad, sino la operación, el procedimiento eficaz, siendo el verdadero fin y función de la ciencia el obrar y trabajar, los descubrimientos para un mejor equipamiento y ayuda en la vida; así, considerar a los hombres como capital se convierte en una simple instrumentalización que contribuye a elevar los niveles de explotación de la fuerza de trabajo, lo cual implica, que entre mayor sea la ilustración de los seres humanos, al poner esta ilustración al servicio de capital, como una nueva forma de capital más avanzada, se está haciendo más intensa la explotación del proceso de cosificación del trabajo resultante de su tratamiento como capital, el mismo que se compra y se vende en el mercado, como cualquier otra mercancía; que tiene como finalidad última la explotación del trabajo de los otros.

A partir de la concepción sobre la necesidad de destinar recursos a la inversión en seres humanos y como un aporte de la teoría del crecimiento, en su libro, Invirtiendo en la Gente, con el cual obtuvo el Premio Nobel de Economía en el año 1979, refiriéndose a una gran mayoría de la población sometida al yugo de la pobreza, Schultz plantea:

"Los factores de producción decisivos para el mejoramiento del bienestar de los pobres no son el espacio, la energía y la disponibilidad de tierra cultivable; los factores decisivos son el mejoramiento de la calidad de la población y los adelantos en el conocimiento."

Con relación a la importancia que se le dio a la tierra como factor fundamental en los procesos de producción de alimentos y a las diferencias en productividad de los suelos como elementos que explican los niveles de pobreza en la gente, Schultz (1985) consideraba como lo más importante para las tierras de cultivo, los incentivos y las oportunidades asociadas que tienen los cultivadores para aumentar la cantidad efectiva de tierra mediante inversiones que incluyen las contribuciones de la investigación agrícola y el mejoramiento de las habilidades humanas.

También planteó como parte integral de la modernización de las economías de países de alto y bajo nivel de renta, el declive de la importancia económica de la tierra de cultivo y el ascenso en importancia del capital humano; por lo cual, el agente humano es considerado el factor crítico en la cuestión de la pobreza, y afirma 
que la inversión en el mejoramiento de la calidad de la población puede aumentar significativamente las perspectivas económicas y de bienestar de los pobres, la atención a los niños, la experiencia laboral y doméstica, la adquisición de información y de habilidades, mediante la escolarización y otras formas de inversión en sanidad y escolaridad, pueden aumentar la calidad de la población.

Considerar el mejoramiento de la calidad de la población y los adelantos del conocimiento como factores decisivos para la producción representa ingresar en lo que paraAdorno y Horkheimer (2007) "constituye la alegoría premonitoria de la dialéctica de la ilustración. Así como la sustituibilidad es la medida del dominio y el más fuerte es aquel que puede hacerse representar en el mayor número de operaciones, del mismo modo la sustituibilidad es el vehículo del progreso y a la vez de la regresión"; donde el supuesto mejoramiento de la población representado sólo en su capacidad de obtener una mayor cantidad de producto por unidad de trabajo o de generar nuevos productos, se convierte en elemento que impulsa el avance y el retroceso social, toda vez que este proceso de mejoramiento de los sistemas de producción a la vez que liberan al hombre de pesadas cargas, también liberan de su trabajo en sentido negativo, al ser reemplazado por la maquina y no poder gozar del trabajo, porque este se cumple bajo la constricción, sin esperanza, con los sentidos violentamente obstruidos, porque la evolución de la maquina se ha convertido en la evolución de la maquinaria del dominio, debido a que los cambios técnicos y sociales se encuentran entrelazados y convergen en la dominación del hombre, resultado de la adaptación al poder del progreso, lo cual a su vez implica el progreso del poder, en un continuo en el cual el ser humano en la medida que logra mayores avances en los sistema de ciencia y tecnología y los pone al servicio de la producción, contribuye al incremento del poder de quienes lo han sometido por medio de las fuerzas del mercado.

De esta manera, su capacidad de trabajo ha sido convertida en una mercancía que se compra y se vende, remunerándola según las leyes de la oferta y la demanda, que han implicado que cada vez se requiera una mayor preparación para el ejercicio de una profesión, mientras la remuneración camina en sentido contrario, cada día se requieren mayores niveles de educación para acceder a los puestos de trabajo, pero esto no se ve compensado con mejoras sustantivas en la calidad de vida de los trabajadores; reduciéndolos a meros objetos de la administración; en esta perspectiva, la búsqueda del mejoramiento de los seres humanos no obedece a una racionalidad sustantiva, sino a una racionalidad instrumental, marcada por la necesidad de mejorar los indicadores económicos y empresariales.

Considerar como mejoramiento de la población la adquisición de información y de habilidades, mediante la escolarización y otras formas de inversión en sanidad y escolaridad, es desconocer el proceso histórico por medio del cual la supervivencia del hombre depende de su capacidad de adaptación a las nuevas condiciones que impone la sociedad; para sobrevivir el hombre es convertido en un aparato que en cualquier momento responde de una forma adecuada a las situaciones perturbadoras y difíciles que conforman la vida, todos tienen que estar preparados para hacer frente a cualquier situación.

Esto mismo sucede con los desarrollos de los procesos de producción, el hombre se adapta, bien sea por medio de la formación para el trabajo o con las diferentes formas de escolarización y sanidad a las nuevas condiciones del mercado de trabajo resultantes de la transformación de los medios de producción material, la aparición del industrialismo ha dado lugar a fenómenos cualitativamente nuevos, a los cuales el trabajador contemporáneo se ha tenido que adaptar de una manera deliberada, acudiendo a las diferentes formas de adecuar su capacidad de trabajo a las condiciones impuestas por los desarrollos de la ciencia y la tecnología implementados en los procesos de producción de bienes y servicios.

Esto es lo que Horkheimer (2002) ha considerado como la tendencia en la vida de hoy a someterse a la racionalización y a la planificación, en la que la vida de todo individuo, incluyendo sus impulsos más secretos, que antes conformaban su esfera privada, tienen que adecuarse a las exigencias de la racionalización y de la planificación: la autoconservación del individuo presupone su adecuación a las exigencias de la conservación del sistema.

Por lo tanto, los llamados mejoramientos de la población vistos como elementos que contribuyen a elevar la productividad y la competitividad de las organizaciones, no son más que los esfuerzos encaminados a 
mantener la vigencia de su fuerza de trabajo para poder ser vendida en los mercados, que cada día, como resultado del avance en la aplicación de los beneficios de la ilustración tienen la tendencia a sacar al individuo de la corriente económica, obligándolo a someterse a una lucha permanente por mejorar su empleabilidad, por medio de las diferentes actividades de capacitación. De otro lado, la conservación y mejoramiento del estado de salud de la población, no se da como una preocupación autentica por mejorar sus condiciones de vida, sino con el objetivo último de hacerlos sujetos de explotación en el mercado laboral; por lo cual se puede asegurar que esta preocupación por el mejoramiento de las personas planteado desde la teoría del capital humano, obedece más a una racionalidad instrumental que a una racionalidad sustantiva enfocad en lograr avances reales a favor de la humanidad.

Otro factor a ser tenido en cuenta en este proceso de supuesto mejoramiento de la calidad de la población, está relacionado con la necesidad de una mayor flexibilidad exigida por el modelo actual de producción, reflejada en la búsqueda de una mayor capacidad de adaptación a circunstancias cambiantes; lo que implica el desarrollo por parte del trabajador de una gran facilidad para pasar de una actividad a otra, sin que esto signifique dedicar mucho tiempo a la reflexión o la necesidad de someterse a extensos procesos de entrenamiento.

Todo esto debido a la uniformidad, cada vez mayor, de los procesos técnicos que facilitan el cambio de ocupación; estos desarrollos en la técnica y la tecnología se ven reflejados en el invento de nuevos aparatos para el dominio de la naturaleza, dando como resultado un mayor sometimiento del hombre a los instrumentos que fueron desarrollados para permitirle dominar la naturaleza, resultando así el mismo hombre dominado por aquello que le debería permitir convertirse en dominador.

Continuando con los planteamientos de Schultz (1985)la calidad de la población es analizada como un recurso escaso, lo que implica que tiene un valor económico y que su adquisición impone un coste; además trató como clave en el análisis del comportamiento humano que determina el tipo y el monto de calidad adquirida a lo largo del tiempo, la relación entre las ganancias obtenidas de la calidad adicional y el coste de adquirirla, así, cuando las ganancias excedan al coste, la calidad de población irá en aumento; esto significa que un incremento en la oferta de cualquier componente de la calidad es una respuesta a la demanda de ese componente.

Con este planteamiento, se ratifica la mercantilización del ser humano en esta teoría, considerar que la población como un recurso escaso con valor económico, implica asimilarlo a cualquier producto, cuya oferta y demanda varía según se tenga una mayor o menor demanda de este tipo de bien, de esta forma se está negando el sí mismo que a cada hombre se le ha dado como suyo propio, que lo hace distinto de todos los demás.

La mirada del hombre como un recurso escaso, con valor económico implica que es igual a todos los demás, en este caso se llega a lo que Adorno y Horkheimer (2007) plantean en la Dialéctica de la Ilustración, como la falta de asimilación del sí mismo por parte de la ilustración que lleva a la coacción social, incluso en los periodos liberales, dándose la unidad del colectivo manipulado y la negación de cada individuo particular; de esta forma, las empresas están interesadas en los hombres solo como clientes y empleados suyos, reduciendo a la humanidad en general y a cada uno de sus elementos en particular a esta fórmula que todo lo agota.

En cuanto empleados, se les llama la atención sobre la organización racional y se les exhorta a incorporarse a ella con sano sentido común, dando lo mejor de sí para lograr los altos niveles de productividad que la actual racionalidad económica impone, por esto se da la necesidad de contar con seres humanos que cada día se ven involucrados en la carrera de mejorar su propia competitividad por medio de procesos de formación, los cuales a su vez se convierten en elementos de explotación, siendo el hombre ilustrado un apéndice más de las nuevas maquinas resultantes del desarrollo de las tecnologías de la información y la comunicación; en este sistema se busca mejorar la calidad de los seres humanos, pero no como tal, sino como seres económicos que contribuyen a elevar las ganancias de las diferentes empresas; que son sujetos de explotación por parte del capital. Como clientes, en cambio, se les presenta a través de episodios humanos privados, en la pantalla o en la prensa, de la libertad de elección y la atracción de lo que no ha sido aún clasificado. En cualquiera de los 
casos, los hombres no dejan de ser objetos, que deben contribuir al logro de los fines de la racionalidad económica.

Desde este enfoque, se cae en la cultura de la autoconservación por la autoconservación, lo que genera la mutación dialéctica del principio del dominio que lleva a la conversión del hombre en instrumento precisamente de esa naturaleza que sojuzga; lo que se representa en una especie de darwinismo social descrito como la sustitución progresiva de la selección natural por la acción racional; en este caso, la supervivencia del individuo depende de su capacidad de adaptación a las coacciones que le impone la sociedad.

Según el planteamiento de la teoría del capital humano, la supervivencia del hombre está determinada por su capacidad de adecuar sus conocimientos y habilidades a las condiciones que le impone el avance de la ciencia, quien no adecua permanente su capacidad para operar en los nuevos escenarios del conocimiento, está condenado a desaparecer; así, para sobrevivir, el hombre es transformado en un aparato que en cualquier momento responde con la reacción adecuada a las situaciones perturbadoras y difíciles que confrontan su vida.

Todos tienen que estar preparados para hacer frente a cualquier situación. Y estar preparado significa desde la perspectiva del capital humano, disponer de un estado de salud que le permita incorporarse al sistema productivo en cualquier momento, además mantener un nivel de conocimientos a la altura de las nuevas circunstancias; esa preparación hace que la educación y la formación para el trabajo se conviertan en elementos de cosificación del hombre al dedicarse más a la preparación de individuos que respondan de manera adecuada a los requerimientos del aparato productivo, sin importar que efectos se puedan lograr en el mejoramiento de los seres humanos visto desde la perspectiva de la racionalidad sustantiva.

Esa necesidad constante de adaptación del hombre a las circunstancias cambiantes del aparato productivo llevan a lo que Horkheimer (2002) llama la tendencia de la vida a someterse cada vez más a la racionalización y la planificación, en la cual, la vida de todo individuo tiende a adecuarse a las exigencias de la racionalización y la planificación; donde la autoconservación del individuo presupone su adecuación a las exigencias de la conservación del sistema, siendo este más importante que el mismo individuo; la masa de los sujetos tiene que adaptarse deliberadamente; el sujeto tiene que emplear todas sus energías para estar en el movimiento de las cosas y pertenecer a ellas.

La adecuación se convierte en pauta para todo signo imaginable de comportamiento subjetivo. El triunfo de la razón subjetiva, formalizada, es también el triunfo de una realidad que se enfrenta arrolladoramente al sujeto como absoluta; esta es la misma realidad que se presenta cuando la teoría del capital humano clasifica a los hombres entre los que han sido sujetos de inversión para convertirlos en capital humano y que por lo tanto son objeto de autoconservación y explotación, mientras los que no se han convertido en esta forma de capital están llamados a la desaparición y son expulsados del sistema productivo formal.

Continuando con el trabajo de Schultz y enfatizando en la importancia de la productividad creciente del capital para los procesos de crecimiento de la economía, Becker (1997), considera la oferta de factores como uno de los aspectos más difíciles y con mayores incertidumbres en la teoría económica; referencia los planteamientos de la economía clásica que identifica tres factores: capital, tierra y trabajo; afirma, que los grandes desarrollos resultantes del esfuerzo del hombre por mejorar la oferta económica de factores de la producción ha llevado a que la tierra sea considerada como un tipo de capital; asimismo, el trabajo con productividad creciente se está tratando como capital; por esta razón, en las economías avanzadas, los sueldos y salarios que se pagan por los trabajos que requieren el uso de la fuerza bruta son muy pequeños en proporción a los que se pagan por aquellos que requieren de inversión en seres humanos para poder ser desarrollados. Sin embargo, considera que el análisis del capital humano no se ha integrado directamente a los estudios generales sobre el capital, debido a que algunos de los determinantes de la oferta de este tipo de capital son únicos, tales como: las influencias sobre la población, la participación de la fuerza de trabajo y la selección de ocupaciones. 
Con relación a la participación de la fuerza de trabajo, considera el volumen total de población como el determinante de las horas de trabajo disponible; las mismas que pueden estar dedicadas a desarrollar actividades que rinden pagos monetarios, también llamadas actividades de mercado; o además, pueden enfocarse a realizar actividades que directamente rinden satisfacción, siendo éstas actividades fuera del mercado y de consumo; el tiempo que no se trabaja, usualmente se llama ocio; pero de hecho, incluye muchas actividades que no son consideradas parte del ocio, como el cuidado de los niños, la limpieza del hogar entre otras.

Para el análisis de la selección de ocupaciones, interpreta las horas trabajadas, como el tiempo dedicado a actividades de mercado, y cada una de estas actividades se llama ocupación; el ejercicio de algunas ocupaciones requiere mayor capacitación que otras; en aquellas que se requiere mayor especialización, la tasa de remuneración debe ser mayor; por lo tanto, la fuente más importante de beneficio de la especialización es la educación y la capacitación, que aumentan la productividad en una ocupación. Se conoce bien el monto de escolaridad que necesitan los médicos, los abogados o los físicos, pero también es importante la capacitación informal que se adquiere en el trabajo, en muchas ocupaciones.

Las influencias sobre la población son todas aquellas actividades que pueden aumentar o disminuir la mortalidad, la productividad, la salud y demás variables demográficas; en este enfoque, la educación reduce la mortalidad e incrementa el estado de salud, porque eleva la productividad de la función de producción del individuo.

En este planteamiento de Becker, se presenta lo que Adorno y Horkheimer (2007) han llamado la eliminación de las cualidades del individuo, su conversión en funciones por medio de la racionalización de las formas de trabajo, en la cual a través de la mediación de la sociedad total, que invade todas las relaciones y todos los impulsos, los hombres son reducidos de nuevo a aquello contra lo cual se había vuelto la ley del desarrollo de la sociedad, el principio del sí mismo, a simples seres genéricos, iguales entre sí; que tienen importancia sólo como poseedores de horas de trabajo que suman al de otros hombres para convertirse en horas de trabajo disponible; de acuerdo con esto, la inversión en el mejoramiento de los seres humanos, está al servicio de diversos intereses económicos, se convierte en un instrumento para todas las empresas y para el sistema económico en general, además producen un fenómeno de homogenización de los seres humanos, que en la búsqueda de permanecer vigentes para ser incorporados a los procesos de producción se convierten en seres genéricos; objeto de explotación por parte del sistema económico y asimilables a la formación de capital.

Complementando los trabajos de Schultz, Solow y Lewis sobre crecimiento económico, Lucas (1996), partiendo de la idea de familias productoras, desarrolló un modelo de crecimiento endógeno de dos sectores; en uno de ellos, la producción final se obtiene por medio de la combinación de capital físico y humano; el producto final puede ser consumido o transformado en capital físico; en el otro sector, la producción y acumulación de capital humano se hace a partir del capital físico y humano. Se considera, además, que la tecnología para la obtención de capital humano es diferente de la que se emplea para la obtención de la producción final.

Según esta teoría, considerar el trabajo como capital humano, trae como consecuencia convertirlo en un factor susceptible de acumular, lo cual constituye una forma de introducir la tecnología en el análisis del crecimiento; sin embargo, uno de los supuestos implícitos, se apoya en el hecho de que el capital físico y el humano son bienes similares, en el sentido de que ambos pueden ser acumulados, a partir de unidades de producción retiradas del consumo. Sin embargo, se puede argumentar que los capitales físico y humano son bienes con unas propiedades enteramente diferenciadas; en particular, la función de producción de capital físico es distinta de la de capital humano, es decir, del proceso de educación, requiere relativamente más capital humano que la producción de capital físico; en otras palabras, la educación es más intensiva en capital humano. Otra distinción fundamental entre capital físico y humano, es que para acumular el segundo, el individuo debe emplear su propio tiempo, mientras que el primero se puede comprar, regalar, o heredar sin necesidad de un esfuerzo propio. 
En el modelo desarrollado por Lucas (1996) la función de producción presenta rendimientos constantes de escala respecto del capital físico y humano, ya que doblar, estas dos formas de capital, conlleva a doblar la producción. Igualmente, trató como una externalidad del stock medio de capital humano, el hecho, de que la gente es más productiva cuando está rodeada de individuos inteligentes y productivos; la existencia de esta externalidad no es esencial para que el modelo genere crecimiento endógeno. También afirmó que los individuos no desean dedicar todo su tiempo a trabajar en la producción de bienes finales, porque prefieren dedicar parte de su tiempo a aumentar su capital humano, es decir, a estudiar; supone que en la producción de capital humano se emplea esta forma de capital como único factor de producción y, además, que existen rendimientos constantes de escala, por lo cual, el aumento neto del stock de capital humano per cápita es igual a la producción de capital humano menos la depreciación, teniendo en cuenta que sí todos los demás factores son constantes, los aumentos de población reducen la cantidad de capital humano disponible por persona.

En la perspectiva de Lucas(1996) el pensamiento y la inteligencia se convierten en elementos a ser utilizados en el proceso de producción, sin dejarle espacio a las formas de contemplación teórica, alejada de los cálculos útiles relacionados con las interacciones del mercado, lo cual no deja espacio para estimular la comprensión de la naturaleza en y por ella misma. En la actualidad la inteligencia esta llamada a desarrollar un papel pragmático para la dominación de la naturaleza en beneficio de las leyes del mercado, la producción de capital humano, no es el resultado del ejercicio de la aplicación de la capacidad de pensamiento a la transformación y la dominación del mundo, por el deseo de conocer y dominar, sino que responde a la necesidad de dominar la naturaleza para obtener de este dominio el lucro que proporciona le explotación de los hallazgos al ser vendido sus frutos en el mercado, así, la misma inteligencia del hombre se convierte en un elemento que debe ser usado por la racionalidad instrumental para producir objetos que el mercado acepte y pague por ellos.

En esta forma de ver al individuo, el principio del sí mismo que se esfuerza por vencer en la lucha contra la naturaleza en general y sobre sus propios impulsos, el yo percibido como algo que está vinculado a funciones de dominio, mando y organización está ausente; sólo el individuo es importante como capital humano que al igual que las maquinas y la tierra está en capacidad de producir bienes que al ser vendidos en el mercado generando unos excedentes.

De esta forma, los hombres al igual que la naturaleza son considerados un mero instrumento, el objeto de una explotación total, que no conoce objetivo alguno puesto por la razón y, por lo tanto, ningún límite, las apetencias son ilimitadas; esto se convierte en la clave para las diversas categorías y métodos de la investigación científica a cuya luz la naturaleza y el propio hombre convertido en capital humano, aparece cada vez más bajo el aspecto de la explotación más eficaz. Esto ha determinado también el modo como los hombres se hacen una imagen unos de otros en sus relaciones políticas y económicas; los modelos de acuerdo con los que la humanidad contempla la naturaleza repercuten finalmente sobre el reflejo del hombre en el espíritu humano, lo determina y excluyen la última meta objetiva que pudiera motivar el proceso.

Retomando las teorías anteriores sobre capital humano, pero dándole un enfoque diferente, en su libro Desarrollo y Libertad, el Premio Nobel de Economía Amartya Sen, aborda el tema y lo relaciona con el de capacidad humana como expresión de libertad; considera muy restringida la perspectiva del capital humano como valor indirecto que puede utilizarse en la producción de bienes y servicios.

Según la perspectiva de Sen (2000) los análisis económicos actuales han tomado en gran medida la acumulación de capital en términos físicos y se concibe como un proceso en el que interviene la calidad productiva de los seres humanos; se aparta de la literatura sobre capital humano que según sus planteamientos tiende a centrar la atención en la capacidad de agencia de los seres humanos para aumentar sus posibilidades de producción; por el contrario, su perspectiva de capacidad humana centra la atención en la capacidad de los individuos para vivir la vida que tienen razones para valorar y para aumentar las opciones reales entre las que pueden elegir. En este sentido, si la educación eleva la eficiencia de las personas en la producción de bienes y servicios, se trata de una mejora en el capital humano, que puede aumentar el valor los bienes económicos, así como el nivel de ingresos de la persona educada; pero al mismo tiempo, le 
proporciona a la persona otros beneficios, como poder leer, comunicarse y desarrollar otras actividades que contribuyen a hacerlo más humano.

Entonces, desde la perspectiva de Sen (2000) se puede decir que los beneficios de la educación son superiores a su papel como capital humano en la producción de bienes; "Si una persona, mejorando su educación, su salud, etc., puede ser más productiva en la fabricación de bienes, no es ilógico esperar que gracias a estos medios también tenga más posibilidades - y libertad-para llevar su vida.”

De acuerdo con este planteamiento Sen (2000) se refiere a una nueva categoría resultante del proceso de mejoramiento de los seres humanos, como resultado de lo que hasta el momento se ha denominado acumulación de capital humano, y lo eleva a la condición de capacidad humana, que además, de servir para lograr incrementos en el crecimiento económico, debe contribuir a mejorar las condiciones de vida de los seres humanos y a la construcción de una sociedad mejor.

No obstante, todos los planteamientos anteriores sobre la importancia de la acumulación de capital humano como factor que contribuye o debería contribuir bien a elevar el rendimiento económico del trabajo y, por lo tanto, prestar una gran contribución al crecimiento de la economía, según algunos teóricos, o a elevar la calidad de vida de los seres humanos según otros; Rifkin (1996) hace un llamado de atención sobre los peligros que amenazan a la humanidad, fruto de los grandes desarrollos de la tecnología que a finales del siglo XX e inicios del XXI, están llevando a un desplazamiento sin precedentes de la capacidad de trabajo del hombre, incluyendo la que se sustenta en el desarrollo de capacidades mentales.

En palabras de Rifkin (1996) si las primeras tecnologías reemplazaban la capacidad física de trabajo humano, sustituyendo cuerpos y brazos por máquinas, las nuevas tecnologías basadas en ordenadores, prometen la sustitución de la propia mente humana, poniendo máquinas pensantes allí donde existían seres humanos, en cualquiera de los ámbitos existentes en la actividad económica. En estos términos, las tecnologías de la información y las telecomunicaciones, emplearán un creciente número de científicos especialistas en ordenadores, ingenieros; productores; escritores; especialistas varios para programar, monitorear y hacer funcionar las redes; sin embargo, dicho número no será tan relevante, si lo comparamos con los millones de empleos que desaparecerán en otros sectores.

Continúa Rifkin (1996) su análisis prospectivo sobre el trabajo, en el cual sólo vislumbra alguna perspectiva favorable para el sector del conocimiento, conformado por una élite de industrias y de disciplinas profesionales responsables de la introducción en la nueva economía de la alta tecnología del futuro, lo que ha llevado al gobierno de los Estados Unidos a plantear la necesidad de reeducar la población para enfrentar los retos de la nueva economía, pero un número importante de críticos no ven importancia ni aplicabilidad a los programas de reeducación, porque la gran mayoría de los trabajadores especializados no podrán alcanzar los niveles que les permitan adecuar su capacidad a las limitadas oportunidades del empleo existente en el sector del conocimiento.

En estos términos, sería necesario preguntarse: ¿la acumulación de capital humano, teniendo al hombre como principal elemento de esa acumulación, seguirá siendo un factor que contribuya a generar crecimiento económico?, O si por el contrario, ¿también en esta función será desplazado el hombre por la máquina, originando una nueva crisis en la economía por falta de consumidores?, ó, ¿qué nuevo sentido tendrá la acumulación de capacidades y conocimientos, si estos no pueden ser aplicados al proceso de producción de bienes y servicios?. ¿Qué sentido tienen los procesos educativos si sólo estáenfocado al mejoramiento de la economía? Las respuestas a estas preguntas no son objeto de este trabajo, pero es importante dejar planteados estos interrogantes.

En este mismo sentido, pero haciendo una alusión más directa, Utria (2002) como parte de su análisis sobre el advenimiento de la internacionalización del neoliberalismo económico y su estrategia de debilitamiento de los países periféricos y el endiosamiento del mercado, hace una crítica a la cultura de consumismo irracional impulsada por el capitalismo salvaje, que ha llevado a "la reducción del hombre y la mujer a la triste condición de capital humano", perdiéndose la amplia gama de círculos grupales y su variedad etnocultural, 
destrezas y especialidades profesionales, sentimientos y aspiraciones, compromisos individuales y colectivos de toda índole, entre otras posibilidades que puede desarrollar el ser humano en beneficio propio y de toda la comunidad.

Otro planteamiento similar hace Aktouf (2001) quien critica la caracterización de las personas como un recurso en las organizaciones, expresa que deben ser tratados como asociados, que pueden hacer escogencia de adherirse, con pleno conocimiento de causa, lo que supone una cierta libertad y responsabilidad; encontramos, entonces, en estas posiciones una forma diferente de abordar el tema del capital humano.

\section{Procedimiento Metodologico}

Se trata de una investigación cualitativa, basada en un proceso de carácter interpretativo y comprensivo de tipo Histórico Hermenéutico, para la cual se uso un diseño investigativo, conformado por cinco componentes, como son:

a) La pregunta objeto de estudio; b) Proposiciones o hipótesis a demostrar; c) La unidad de análisis; d) La unión lógica entre los datos correspondientes a las variables; e) El criterio para interpretar los hallazgos o descubrimientos.

La investigación busca comprender el hecho económico de la formación o acumulación del Capital Humano y su relación con el crecimiento económico. El método utilizado responde a una finalidad de descripción, interpretación, argumentación, que permitan avanzar hacia la comprensión de las temáticas estudiadas en un proceso dialéctico para lo cual se hace un abordaje crítico desde los postulados de la Escuela de Frankfurt. Para el desarrollo de la investigación se avanza en las siguientes fases o etapas:

- Fase descriptiva. A partir del interrogante planteado en la formulación del problema, se busca identificar las diferentes concepciones, enfoques y tendencias teóricas representativas para determinar el avance y el estado actual de la teoría económica en los temas de Capital Humano, y crecimiento económico, esto se complementa con una visión crítica desde los postulados de la Escuela de Frankfurt, además se realiza una doble contingencia considerando visiones contrarias a la teoría del capital humano planteadas desde la propia ciencia económica; en términos de argumentos, procesos de reflexión y reconocimiento de actores; de igual forma se establece el estado actual de las reflexiones de los diferentes estudiosos sobre estos temas.

- Fase interpretativa. Con base en las diferentes concepciones, argumentos, enfoques y tendencias teóricas identificadas en la fase descriptiva, se plantea la relación entre las variables, y se realiza el proceso de interpretación, lo que lleva a la formulación de los argumentos interpretativos; después de validados estos se pasa a la fase siguiente.

- Fase de construcción de sentido o construcción teórica. A partir de los argumentos interpretativos se identifican los hilos conductores, lo que permite la formulación de los argumentos de sentido. Con base en la información de teoría económica y de los planteamientos de la Escuela de Frankfurt, resultante de los estudios referenciados en el trabajo y el estado del arte, se hace la evaluación sobre las variables de estudio. Con base en estos argumentos y su interpretación se obtienen las siguientes conclusiones. 


\section{Conclusiones}

En este análisis de la teoría del capital humano, se puede ver como el proceso histórico de evolución de la teoría, ha estado marcado por una mirada funcionalista con una preocupación por la productividad y la eficacia de las organizaciones, que se puede lograr por medio de los llamados procesos de mejoramiento de los seres humanos, bajo esta mirada, la formación, la educación y el mejoramiento de los estados de salud de la población se convierten en instrumentos de las organizaciones para lograr fines en el mercado, se piensa en el mejoramiento de los seres humanos, solo como una función del mercado, dejando de lado la importancia que esto puede tener para la sociedad.

El enfoque observado permite concluir que la teoría del capital humano se ha centrado en el mercado y la economía como un todo, sin tener en cuenta que el mercado es uno de los muchos enclaves de la sociedad y no el único; lo cual ha convertido a esta teoría en una racionalidad instrumental basada en medios y fines, donde el ser humano se convierte en el medio por el cual el capitalista, además de convertir su dinero en capital, logra transformar la calidad del trabajo por medio de la educación, la formación para el trabajo y el mejoramiento del estado de salud de los empleados; pero esta transformación de la calidad del trabajo no responde a la necesidad de mejorar las condiciones de la sociedad, sino a un interés de lucro, el cual se ve incrementado por medio del crecimiento de la productividad del trabajo que se logra cuando su portador ha sido sometido a procesos que le permiten la ejecución de tareas que requieren altos niveles de formación; así, las políticas públicas orientadas a lograr mejoras en los sistemas de salud y de educación, no son pensados en términos de mejoramiento de la sociedad y de incremento de la libertad de los seres humanos, sino en términos de mejoramientos del mercado de trabajo y en una mayor productividad de las empresas; por lo tanto el ser humano se convierte en un medio para lograr un fin, relacionado con la actividad económica.

Bajo esta racionalidad instrumental, los avances en ciencia y tecnología son elementos que hacen necesaria la inversión en seres humanos para incrementar el llamado capital humano; que visto como tal,no contribuye al mejoramiento de la sociedad; sino al proceso de adaptación de los seres humanos a las nuevas condiciones que el mercado laboral impone, como consecuencia de la aplicación de los adelantos tecnológicos a los procesos de producción de bienes y servicios; de esta forma, la inversión en lo que se ha llamado el mejoramiento de los seres humanos, no representa un verdadero avance en ese mejoramiento, es sólo una adecuación a las nuevas condiciones de los sistemas de producción, lo que lleva al hombre a una búsqueda permanente por mantener su capacidad de producción acorde con las condiciones del mercado; de esta forma, la educación, la formación para el trabajo y el mantenimiento del estado de salud, dejan de ser un factor de ventaja en el mercado laboral y se convierten en una obligación para poder mantener el nivel de competencia que el mercado ha impuesto gracias a la aplicación de los avances de la ciencia a los procesos de producción; como resultado, la adquisición de nuevos conocimientos se convierte en el nuevo dios que hay que buscar de manera permanente de acuerdo con las condiciones cambiantes y los mayores niveles de competitividad, para no ser expulsado del mercado laboral.

La existencia del mercado laboral en el cual se intercambian servicios personales, es el resultado de la cosificación y la conversión del trabajo en una mercancía, que se compra y se vende de acuerdo con los valores de uso y los valores de cambio contenidos en ella, y que son objeto de cambio solo en la medida que puedan producir otras mercancías demandadas en el mercado de bienes y servicios; en la medida que se incrementa el nivel de educación de una persona, se considera que está aumentando su capital humano, por la posibilidad de generar bienes y servicios que adquieren un mayor precio en el mercado y por lo tanto deben aumentarla ganancia pecuniaria de su poseedor y de quien la compra para incorporarla en el proceso de producción de otras mercancías; en la medida que para su producción sea necesario tener en cuenta mayores niveles de capacitación de sus productores, se desconoce la importancia de la educación como generador de capacidad de pensamiento que permite distinguir al hombre del animal, haciendo al hombre humano; este pensamiento que se podría dedicar a la producción de arte o ciencia orientadas a engrandecer más al hombre, se dedican a la producción de mercancías que tienen valor para el mercado, negándose de esta formala esencia del hombre, al hacer importante el pensamiento sólo en la medida que pueda estar enfocado a la producción de cosas aceptadas por el mercado. 
Los desarrollos de la ciencia y la tecnología que deberían estar más orientados a contribuir a la libertad del hombre, han generado el proceso contrario; limitando la libertad, en la medida que considerar al hombre como capital humano lo vincula al proceso de producción y lo hace dependiente de este proceso, pues su capacidad de pensamiento solo es útil si puede ser incorporado a la producción de bienes y servicios, en este caso, la falta de libertad esta determinada por convertir al hombre en otra cosa diferente de su esencia, en una mercancía que se compra y se vende. De esta forma, el intelectual no esta libre de las presiones de la economía mercantil para vincularse al mercado laboral y dedicar su capacidad a elevar los niveles de productividad de las organizaciones, que están insertas en un mundo cada vez más competitivo; así, el intelectual se ve sometido por el mercado y pierde su libertad de pensamiento, al enajenarla en beneficio de la racionalidad instrumental contenida en los procesos de producción. Visto el hombre como capital humano, su pensamiento se desarrolla según un proyecto de producción, lo cual implica una racionalidad sujeta a una finalidad, lo que limita la posibilidad de su auto desarrollo.

Bajo la teoría del capital humano se considera al trabajador con conocimiento como el activo más valioso de una organización; entendiendo por tal a un ejecutivo con conocimiento, que sabe cómo asignar conocimiento a usos productivos, justamente como el capitalista sabe como asignar capital a usos productivos; si el conocimiento está en el cerebro de las personas, se hace necesario lograr el compromiso personal de los empleados y su identidad con la empresa, su visión, su misión y sus valores; a este respecto la creación de conocimiento tiene que ver tanto con ideas como con ideales, lo cual sirve como incentivo para la innovación; de esta forma, el trabajador cualquiera sea su condición, es tratado como un recurso y solo se le da importancia en la medida que su conocimiento puede ser asignado a usos productivos; en la búsqueda del compromiso para poder lograr sacar el máximo provecho de su conocimiento se consuma la cosificación de la persona y la instrumentalización de su conocimiento, por lo cual el ser humano es importante como recurso y no como persona.

El capital humano se considera importante en la medida que puede generar un movimiento productor de conocimiento, del cual participa también la sociedad a través del sistema educativo e investigativo como entorno de las empresas; este conocimiento entra a participar del mundo empresarial, no sólo en forma de máquinas, equipos o materias primas, sino también en el crecimiento de la capacidad para gestionar los procesos administrativos, de producción y comercialización; se transforma en lo que se ha denominado el conocimiento organizacional; entendido como la capacidad de una compañía para generar nuevos conocimientos, diseminarlos entre los miembros de la organización y materializarlos en productos, servicios y sistemas; este tipo de conocimiento representa la base de la innovación y la competitividad de las organizaciones. En este proceso de generar y usar conocimiento en los procesos de producción y lograr una mayor competitividad se observa una hegemonía de la racionalidad instrumental, bajo el signo de la deshumanización de la sociedad reducida a sistema, condicionada a los requerimientos de la lógica económica, del modelo dominante.

En la teoría del capital humano se pierde la identidad de la clase trabajadora como clase y se convierte en un agregado de vendedores de fuerza de trabajo, disfrazando la relación entre sujeto y objeto; entre clase y capital de una manera dialéctica, lo cual impide ver la verdadera relación entre trabajo y capital, como una relación de contrarios, en la cual la clase trabajadora se enfrenta al capital; en el caso de la teoría del capital humano, se pretende convertir al trabajador en su contrario. 


\section{Referencias}

ADORNO, T. W.; HORKHEIMER, M. Dialéctica de la Ilustración. Madrid: Ediciones Akal, S.A, 2007.

AKTOUF, O. La estrategia del avestruz racional. Cali: Universidad del Valle, 2001.

BECKER, G. Teoría Económica. Bogotá: Fondo de Cultura Económica, 1997.

HABERMAS, J. Aclaraciones a la Ética del Discurso. Madrid: Trotta, 2000.

HABERMAS, J. Entre Naturalismo y Religión. Barcelona: Paidós Iberíca S.A, 2006.

HORKHEIMER, M. Crítica de la razon instrumental. Madrid: Trotta, 2002.

LEWIS, W. A. Teoría del Desarrollo Económico. México: Fondo de Cultura Económica, 1976.

LUCAS, R. E. Nobel lecture: Monetary neutraly. Journal of political economy, v. 104, n. 4, p. 661-682, Agosto 1996.

MARX, K. El Capital. Critica de la Economía Política. México: Fondo de Cultura Económica, 1991.

RIFKIN, G. El fin del trabajo. Barcelona: Paidos Ibérica, 1996.

SCHULTZ, T. Invirtiendo en la Gente. Barcelona: Ariel S.A, 1985.

SEN, A. Desarrollo y libertad. Bogotá: Planeta, 2000.

SMITH, A. Investigación de la Naturaleza y Causas de la Riqueza de las Naciones. Barcelona: Orbis S.A., 1983.

UTRIA, R. D. El desarrollo de las naciones: Hacia un nuevo paradigma. Bogotá: Alfaomega, 2002.

WALRAS, L. Elementos de Economía Política Pura. Madrid: Alianza S.A., 1987.

WEBER, M. La ética protestante y el espiritu del capitalismo. México: Fondo de Cultura Económica, 2003. 